\title{
INIIALT
}

F. Stammberger: Die Aufgaben der Geologen im Siebenjahrplan . . . . . . . . . . 517

J. Dockter \& P. PUFF: Neue Kartensymbole für die Gliederung des Unteren und Mittleren Buntsandsteins in Thüringen auf zyklischer Grundlage . . 548

E. LANGE: Rekonstruktion in der UdSSR . . . . . . 520

E. Kautzsch: Die einzelnen Stadien bei der geologischen Erkundung von Lagerstätten nutzbarer mineralischer Rohstoffe . . . . . . . . . . . . . . . 523

H. Wolf \& G. OlszaK: Das Kernresonanzmagnetometer als Prospektionsgerät in der angewandten Geophysik . . . . . . . . . . . . . 554

G. NoSSKE: Eine neue leichte Feldausrüstung für die induzierte Polarisation mit Gleichstromimpulsen . . 528

W. I. Krasnikow: Auffinden tiefliegender Erzlagerstätten durch Untersuchung der primären Dispersionshōfe . . . . . . . . . . . . . . 533

J. Zieschang: Grundsätze komplexer hydrogeologischer Untersuchungen im Bereich der Lockergesteine 535

R. HAAGE: Geologische und petrographische Untersuchungen des Tertiärquarzits von Morl . . . . . . 539

R. Neumann: Zum Einfluß des Kalkgehaltes auf die Korngrößenanalysen mitteldeutscher Löße . . . . . 544

A. Ludwig: Eine Ergänzung zum SM XX „Citoplast“ (VEB Zeiss Jena) für das Auslesen von Mikrofossilien . . . . . . . . . . . . . . 555

H. Dietze: Phtalopal G zum Präparieren dünnschaliger Lamellibranchiaten . . . . . . . . . . . 556 Lesesteine . . . . . . . . . . . . . . 557 Besprechungen und Referate....... . . 558 Nachrichten und Informationen . . . . . . . . . 561 Kurznachrichten ............. 564

Die ZeItschrift FÜr ANGewandte Geologie berichtet ständig ausführlich über folgende Arbeitsgebiete: Geologische Grundlagenforschung und Lagerstättenforschung / Methodik der geologischen Erkundung / Ökonomie und Planung der geologischen Erkundung / Technik der geologischen Erkundung / Geologie und Lagerstättenkunde im Ausland / Bibliographie, Verordnungen, Richtlinien, Konferenzen, Personalnachrichten

Dem Redaktionskollegium gehören an:

Prof. Dipl.-Berging. BÜHRIG, Nordhausen - Prof. Dr. HECK, Schwerin - Prof. Dr. KAUTZSCH, Berlin Prof. Dr. LANGE, Berlin - Dr. MEINHOLD, Leipzig - Dr. NOSSKE, Leipzig - Prof. Dr. PIETZSCH, Freiberg Dr. REH, Jena - Prof. Dr. SCHÜLLER, Berlin - Dipl.-Berging.-Geologe STAMMBERGER, Berlin

Prof. Dr. WATZNAUER, Karl-Marx-Stadt

Chefredakteur: Prof. Dr. ERICH LANGE, Berlin

Die Zeitschrift für ANGewandte Geologie ist kein Organ einer engen Fachgruppe. Auf ihren Seiten können alle strittigen Fragen der praktischen Geologie behandelt werden. Die Autoren tubernehmen für ihre Aufsätze die übliche Verantwortung.

Herausgeber: Staatliche Geologische Kommission und Zentrale Vorratskommission für mineralische Rohstoffe der Deutschen Demokratischen Republik. Chefredakteur: Prof. Dr. Erich Lange, Berlin. Redaktion: Berlin N. 4, Invalidenstraße 44. Verlag: Akademie-Verlag GmbH, Berlin W 1, Leipziger StraBe 3-4 (Fernsprecher 220441, Postscheckkonto: Berlin 35021). Bestellnummer des Heftes: 1047/5/11. Die „Zeitschrift für angewandte Geologie" erscheint monatlich. Bezugspreis 2,- DM je Heft. - Satz und Druck: Druckhaus „Maxim Gorki“", Altenburg. Verőffentlicht unter der Lizenznummer ZLN 5008 des Ministeriums für Kultur, Hauptverwaitung Verlagswesen. Karten: MdI der DDR Nr.5138/K 11. Printed in Germany 


\title{
ZEITSCHRIFT
}

FÜR

CHEFREDAKTEUR: PROF. DR. E. LANGE

\section{ANGEWANDTE GEOLOGIE}

BAND 3 - NOVEMBER 1959 - HEFT 11

\section{Die Aufgaben der Geologen im Siebenjahrplan}

\author{
Friedrich STAMmberger, Berlin
}

Nachfolgend veröffentlichen wir den Schlußteil des Referates von F. STAMMBERGER, Direktor des Zentralen Geologischen Dienstes, „Die Aufgaben der Staatlichen Geologischen Kommission im Siebenjahrplan" auf der Industriezweigkonferenz der StGK am 10. Juli 1959 in Halle.

Die Redaktion

Werte Kollegen und Genossen!

Die Rekonstruktionspläne werden zusammen mit den Volkswirtschaftsplänen in den Arbeitsgruppen eingehend erörtert werden. Zweifellos ist dort der bessere Platz, um alle Positionen allseitig zu prüfen. Die $\mathrm{Zu}$ sammensetzung unserer Industriezweigkonferenz, die Tatsache, daß neben vielen Kollegen aus der unmittelbaren Produktion auch zahlreiche qualifizierte und fachkundige Gäste teilnehmen, ist sichere Gewähr für eine gründliche Behandlung der vorliegenden Materialien.

Gestatten Sie mir daher, auf einige Fragen einzugehen, die gewöhnlich bei solchen umfassenden Konferenzen wie diese etwas zu kurz geraten, ich meine einige geologische Grundfragen und einige kurze Hinweise auf gewisse Arbeitsgebiete, über die nur selten gesprochen wird, obwohl sie äußerst wichtig sind. Ich möchte mit letzteren beginnen.

In unseren Rekonstruktionsplänen der Geologischen Dienste und des Zentralen Geologischen Dienstes (ZGD) nehmen die Ausgaben für die Erweiterung unserer Labors einen beachtlichen Platz ein. Es wird kaum einen unter den Anwesenden geben, der die Notwendigkeit der Durchführung von Analysen bestreitet. Und es gibt wohl kaum einen unter uns, der sich Rechenschaft über den tatsächlichen Umfang dieser Arbeiten ablegt. Nehmen wir nur die chemischen Analysen. 1958 wurden rund 40000 Einzelbestimmungen im Zentral- und in den Außenlabors durchgeführt, fast 33000 spektrochemische Analysen und rund 12000 physiko-technische Bestimmungen. Wenn wir einen solchen Arbeitsumfang bereits $1958 \mathrm{zu}$ bewältigen hatten, dürfte klar sein, da $B$ wir ohne bedeutende Kapazitätserweiterung unserer Labors bei der Durchführung unserer kommenden Arbeiten an diesem Engpaß scheitern könnten.

Nehmen wir einen anderen wenig beachteten Abschnitt unserer Arbeit: die Gutachtertätigkeit im ZGD und in den Diensten. Wir fertigen jährlich insgesamt bis zu 4500 ingenieurgeologische und hydrogeologische Gutachten an, für die wir Gebühren in Höhe von rund 500000-600000 DM vereinnahmen. Gemessen am Gesamtvolumen unseres Budgets ist das nicht imponierend. Wenn wir jedoch den volkswirtschaftlichen Nutzen dieser Gutachten betrachten, wenn wir uns erinnern, da $B$ in der DDR kein irgendwie beachtlicher Bau, keine Wasserversorgungsfrage ohne unsere Mit- arbeit entschieden wird, dann ist leicht zu ermessen, daß sich der Wert dieser Dienstleistungen nicht in der genannten Ziffer ausdrückt. Das ist lediglich eine bescheidene Gebühr, die nebenbei bemerkt in gar keinen Verhältnissen zu den Beträgen steht, die, von staatlichen Stellen und VE-Betrieben für Privatgutachten, zuweilen äußerst fraglicher Qualität, gezahlt werden. Wir sind, wie sich Kollege Dr. HoHL einmal ausdrückte, in dieser Beziehung die billigste $\mathrm{HO}$ der DDR.

Gestatten Sie mir, daB ich all den Kollegen, die in der Stille und meist unbemerkt diese Aufgaben erfüllen, von hier aus den Dank der StGK ausspreche und mit ihm den Wunsch verbinde, daB sie in Zukunft noch engere Verbindungen zu den örtlichen staatlichen Organen herstellen, sie in allen Fragen der Geologie und Hydrogeologie beraten, selbstlos, kameradschaftlich und mit noch größerer Sachkenntnis als bisher.

Kollegen, diese scheinbare Abschweifung schien mir notwendig, weil wir meist bei den Diskussionen über die Fragen der Erkundung von Lagerstätten diesen nicht unwichtigen Teil unserer Arbeit zu erwähnen vergessen.

Gestatten Sie mir, auf eine Grundfrage der geologischen Erkundung einzugehen und dabei eine Behauptung an den Ânfang zu stellen: In der DDR ist heute nicht mehr die geologische Erkundung einer entdeckten Lagerstätte, ihre industrielle Erkundung, die schwierigste Etappe unserer Tätigkeit. Bei uns - wie in allen geologisch gut erforschten Ländern - ist das Schwierigste und Komplizierteste das Auffinden und Entdecken neuer Lagerstätten selbst.

Für die industrielle Erkundung bereits entdeckter Lagerstätten, für ihre sachgemäße Untersuchung und Umgrenzung gibt es recht brauchbare Methoden, die ein durchschnittlich begabter Geologe bei einiger Erfahrung mit bestem Erfolg der Individualität jeder Lagerstätte anpassen kann. Neben diesen methodischen Hinweisen gibt es Instruktionen der ZVK und Arbeitsrichtlinien für die Dokumentation und Probenahme. Die industrielle Erkundung einer Lagerstätte ist heute nicht so sehr ein wissenschaftliches Problem als ein organisatorisches. Das geologisch-technische Projekt formuliert daher die Art und Weise, wie eine Aufgabe in kürzesten Fristen bei geringstem Aufwand am besten und ausreichend für die Bestätigung durch die ZVK zu lösen ist.

Ganz anders steht es mit der Entdeckung neuer Lagerstätten, vor allem bei uns in der DDR. Neue Lagerstätten in der DDR gehören - darüber sind sich die Geologen einig - fast ausschließlich zum Typ der verdeckten, nicht zutage ausgehenden Lagerstätten. 